\title{
EVALUATING SPARSITY PENALTY FUNCTIONS FOR COMBINED COMPRESSED SENSING AND PARALLEL MRI
}

\author{
Daniel S. Weller ${ }^{1}$, Jonathan R. Polimeni ${ }^{2,3}$, Leo Grady ${ }^{4}$, Lawrence L. Wald ${ }^{2,3}$, \\ Elfar Adalsteinsson ${ }^{1}$, Vivek $K_{\text {Goyal }}{ }^{1}$ \\ ${ }^{1}$ Dept. of EECS, Massachusetts Institute of Technology, Cambridge, MA, USA \\ ${ }^{2}$ A. A. Martinos Center, Dept. of Radiology, Massachusetts General Hospital, Charlestown, MA, USA \\ ${ }^{3}$ Dept. of Radiology, Harvard Medical School, Boston, MA, USA \\ ${ }^{4}$ Dept. of Image Analytics and Informatics, Siemens Corporate Research, Princeton, NJ, USA \\ dweller@mit.edu, jonp@nmr.mgh.harvard.edu, leo.grady@ siemens.com,wald@nmr.mgh.harvard.edu, \\ elfar@mit.edu,vgoyal@mit.edu
}

\begin{abstract}
The combination of compressed sensing (CS) and parallel magnetic resonance (MR) imaging enables further scan acceleration via undersampling than previously feasible. While many of these methods incorporate similar styles of CS, there remains significant variation in the particular choice of function used to promote sparsity. Having developed SpRING, a framework for combining CS and GRAPPA, a parallel MR image reconstruction method, we view the choice of penalty function as a design choice rather than a defining feature of the algorithm. For both simulated and real data, we compare different sparsity penalty functions to the empirical distribution of the reference images. Then, we perform reconstructions on uniformly undersampled data using a variety of penalty functions to illustrate the impact appropriately choosing the penalty function has on the performance of SpRING. These experiments demonstrate the importance of choosing an appropriate penalty function and how such a choice may differ between simulated data and real data.
\end{abstract}

Index Terms - Compressed sensing, magnetic resonance imaging, image reconstruction, parallel imaging, sparsity penalty functions

\section{INTRODUCTION}

Long acquisition times continue to limit magnetic resonance (MR) imaging; aggressive undersampling of $k$-space greatly diminishes the cost and duration of acquiring an MR image and enable novel applications. Conventional methods like GRAPPA [1] have had some success in practical applications. Meanwhile, compressed sensing (CS) [2-5] provides a different approach to recovering images from undersampled data, assuming the image is sparse enough, and the undersampling is incoherent enough for the desired number of observations. Since the spatial coil weighting is complementary to image sparsity, there has been much interest in combining these methods to yield further improvements in reconstruction quality and feasible accelerations. In [6,7], parallel MR imaging methods like GRAPPA are combined with CS to reconstruct highly undersampled

Funding acknowledgments: NSF CAREER Grant CCF-0643836, NIH R01 EB007942 and EB006847, NIH NCRR P41 RR014075, and a NSF Graduate Research Fellowship. The authors also thank Prof. Fa-Hsuan Lin of the A. A. Martinos Center for the use of his $B_{1}$ simulator. $k$-space data. One differentiating feature between these implementations (and among many others) is the choice of function used to penalize (lack of) sparsity.

GRAPPA involves using a kernel to compute missing $k$-space frequencies. The kernel is chosen to emulate a shift in frequency (multiplying by a complex exponential in the image domain); when convolved with the known data, the output represents estimates of the missing frequencies. GRAPPA expects the data to be undersampled uniformly; otherwise, multiple kernels can be used, one for each block of known/missing points in $k$-space. GRAPPA estimates these kernels from a small block (called the ACS block) of additionally acquired data near the center of $k$-space. GRAPPA is limited by the noise amplification from applying the kernel to the undersampled data. To mitigate noise amplification, the reconstruction process can be regularized using a penalty like sparsity.

CS assumes a sparsity prior on the image itself to enable its recovery from relatively few samples. In [5], CS is applied to MR imaging, recovering an anatomical image of a brain using the approximate sparsity of its discrete wavelet transform (DWT). This approach uses the $\ell_{1}$-norm to penalize sparsity, but many other approximations to the exact sparsity penalty function, the $\ell_{0}$-"norm," are also used with CS. For example, the Welsch function is used in [8] to reconstruct another brain image. Other penalty functions, like the $\ell_{p}$-"norm," for $p<1$ (see $[9,10]$ ), and the Cauchy function can be used as well. In this paper, we revisit the properties of several different choices of penalty functions, evaluate their suitability for the sparsity of simulated and real data, and explore their effects on the reconstruction algorithm originally derived in [6].

\section{THEORY}

Introduced in [6], the Sparse Reconstruction of Images using the Nullspace method and GRAPPA is called SpRING. The undersampled $k$-space data $\mathbf{d}$ is processed by GRAPPA to produce the full $k$-space GRAPPA result $\mathbf{W}(\mathbf{d})$. The ACS block is also used to form low-resolution estimates of the coil sensitivities for the $P$ coils; let $S_{p}(x, y, z)$ represent the coil sensitivity weighting the voxel at $(x, y, z)$ in the $p$ th coil. Let $m(x, y, z), m_{p}(x, y, z)$, and $\eta_{p}(x, y, z)$ be the object magnetization, $p$ th coil image, and $p$ th coil additive noise at $(x, y, z)$; then $m_{p}(x, y, z)=S_{p}(x, y, z) m(x, y, z)+$ $\eta_{p}(x, y, z)$, where $\left[\eta_{1}(x, y, z) \cdots \eta_{P}(x, y, z)\right]^{T}$ is zero-mean normal with coil noise covariance $\boldsymbol{\Lambda}$. The coil combination weights 
$C_{p}(x, y, z)$ form the linear least squares estimate; following [11],

$$
\left[\begin{array}{c}
C_{1}(x, y, z) \\
\vdots \\
C_{P}(x, y, z)
\end{array}\right]^{T}=\operatorname{pinv}\left(\boldsymbol{\Lambda}^{-1 / 2}\left[\begin{array}{c}
S_{1}(x, y, z) \\
\vdots \\
S_{P}(x, y, z)
\end{array}\right]\right) \boldsymbol{\Lambda}^{-1 / 2}
$$

where $\operatorname{pinv}(\cdot)$ is the left pseudo-inverse.

The SpRING method jointly optimizes for sparsity and fidelity to the GRAPPA solution, operating in the nullspace of the observation matrix $\mathbf{K}$. The coil combination weights are used both in the GRAPPA fidelity term to weight the contributions of each point in $k$-space to the final image and to combine the SpRING result (full $k$-space for each coil) into a single image. Starting with continuation parameter $\alpha=1$, we use IRLS (see [12]) to find the missing data $\mathbf{x}$ that minimizes

$$
\begin{aligned}
\| \mathbf{C F}^{-1}\left(\overline{\mathbf{K}}^{T} \mathbf{x}+\mathbf{K}^{T} \mathbf{d}\right. & -\mathbf{W}(\mathbf{d})) \|_{2}^{2} \\
& +\lambda\left\|\mathbf{\Psi} \mathbf{F}^{-1}\left(\overline{\mathbf{K}}^{T} \mathbf{x}+\mathbf{K}^{T} \mathbf{d}\right)\right\|_{S(\alpha)},
\end{aligned}
$$

where $\mathbf{C}$ is a diagonal matrix of the coil combination weights from (1); $\mathbf{F}^{-1}$ is the inverse DFT; $\mathbf{K}$ and $\overline{\mathbf{K}}$ select the known and missing values, respectively, from full-FOV $k$-space data; $\Psi$ is the sparsifying transform; $\|\cdot\|_{S(\alpha)}$ is the sparsity penalty function, parameterized by $\alpha$; and $\lambda$ is a tuning parameter balancing sparsity against fidelity to the GRAPPA reconstruction. The sparsity penalty function is assumed to be separable, so $\|\mathbf{w}\|_{S(\alpha)}=\sum_{n=1}^{N} s_{\alpha}\left(w_{n}\right)$. To accommodate simultaneous sparsity across $P$ coils, $\|\mathbf{w}\|_{S(\alpha)}=\sum_{n=1}^{N} s_{\alpha}\left(\left\|\left[w_{n, 1} \cdots w_{n, P}\right]\right\|_{2}\right)$. When increased sparsity is desired, this optimization problem is repeated for larger $\alpha$, using the result as a starting point. For this repetition to be viewed as homotopy continuation, starting at an easier problem and progressing closer to the optimization problem we want to solve, we want $\|\cdot\|_{S(\alpha)}$ to be nearly convex when $\alpha$ is small, and $\|\cdot\|_{S(\alpha)} \rightarrow\|\cdot\|_{\ell_{0}}$ as $\alpha \rightarrow \infty$. Since we solve for the missing data, the acquired data is preserved.

Performing IRLS to minimize (2) involves repeatedly computing a diagonal weight matrix $\mathbf{D}_{\alpha}$ from the current value of $\mathbf{x}$ and solving the least-squares problem

$$
\begin{aligned}
& \overline{\mathbf{K}} \mathbf{F}\left(2 \mathbf{C}^{H} \mathbf{C}+\lambda \boldsymbol{\Psi}^{H} \mathbf{D}_{\alpha} \boldsymbol{\Psi}\right) \mathbf{F}^{-1} \overline{\mathbf{K}}^{T} \mathbf{x}= \\
& \overline{\mathbf{K}} \mathbf{F}\left(2 \mathbf{C}^{H} \mathbf{C F}^{-1}\left(\mathbf{W}(\mathbf{d})-\mathbf{K}^{T} \mathbf{d}\right)-\lambda \boldsymbol{\Psi}^{H} \mathbf{D}_{\alpha} \boldsymbol{\Psi} \mathbf{F}^{-1} \mathbf{K}^{T} \mathbf{d}\right) .
\end{aligned}
$$

In general, $\left[\mathbf{D}_{\alpha}(\mathbf{x})\right]_{n, n}=\frac{1}{w_{n}} \frac{\partial\|\mathbf{w}\|_{S(\alpha)}}{\partial w_{n}}$, evaluated at $w_{n}=$ $\left[\mathbf{\Psi} \mathbf{F}^{-1}\left(\overline{\mathbf{K}}^{T} \mathbf{x}+\mathbf{K}^{T} \mathbf{d}\right)\right]_{n}$. Since the difficulty of the least-squares problem depends on the condition number of the total left side, severe ill-conditioning of $\mathbf{D}_{\alpha}$ can be expected to adversely impact the speed and performance of the overall algorithm.

While proposed in [6] with the Welsch function $s_{\alpha}\left(w_{n}\right)=1-$ $e^{-\alpha\left|w_{n}\right|^{2}}$, other penalty functions can be used as well. The $\ell_{1}$ norm $s_{\alpha}\left(w_{n}\right)=\left|w_{n}\right|$ and the Cauchy function $s_{\alpha}\left(w_{n}\right)=\log (1+$ $\left.\alpha\left|w_{n}\right|^{2}\right) / \log (1+\alpha)$ are compatible with the algorithm. Because sparsity mainly concerns distinguishing between very small sparse values from larger non-sparse values, we pay special attention to the behavior of these penalty functions near zero and near infinity. From a Bayesian standpoint, the choice of sparsity penalty function corresponds to a prior on the coefficients in the sparse transform domain.

The Welsch function is nonconvex, bounded, and approaches 1 exponentially fast as $\left|w_{n}\right| \rightarrow \infty$. It is convex near the origin, for $\left|w_{n}\right|^{2}<1 /(2 \alpha)$. The derivative (w.r.t. $\left.w_{n}\right)$ is $2 \alpha w_{n} e^{-\alpha\left|w_{n}\right|^{2}}$.
When $\left|w_{n}\right|$ is small, $1-e^{-\alpha\left|w_{n}\right|^{2}} \approx \alpha\left|w_{n}\right|^{2}$, with derivative $2 \alpha w_{n}$. Since the Welsch function is bounded above, the prior of the form $e^{-s_{\alpha}\left(\left|w_{n}\right|\right)}$ is not integrable over $[0, \infty)$, so the Welsch function corresponds to an improper prior.

The $\ell_{1}$-norm is popular because the CS optimization problem becomes convex. The norm is linear both near zero and for large $\left|w_{n}\right|$, with constant-magnitude derivative $w_{n} /\left|w_{n}\right|$ (except at zero due to a discontinuity). The $\ell_{1}$-norm corresponds to the exponential distribution with scale parameter 1 . One can similarly consider concave $\ell_{p}$-"norm" penalty functions; these functions are considered in depth in [10].

The Cauchy function is nonconvex and increases logarithmically fast. It is convex near the origin (for $\left|w_{n}\right|^{2}<1 / \alpha$ ); the Cauchy function increases slowly (but without bound) for large $w_{n}$. The derivative of the Cauchy function is

$$
\frac{2 \alpha w_{n}}{\log (1+\alpha)\left(1+\alpha\left|w_{n}\right|^{2}\right)}
$$

which is approximately $2 w_{n} /\left(\log (1+\alpha)\left|w_{n}\right|^{2}\right)$ when $w_{n}$ is large. When $\left|w_{n}\right|$ is small, $\log \left(1+\alpha\left|w_{n}\right|^{2}\right) \approx \alpha\left|w_{n}\right|^{2}$, similar to the Welsch function. The Cauchy and Welsch functions can be thought to treat small sparse values like the $\ell_{2}$-norm does and large non-sparse values like the $\ell_{0}$-"norm" does. The prior corresponding to the Cauchy function is proportional to $\exp \{-\log (1+$ $\left.\alpha) s_{\alpha}\left(\left|w_{n}\right|\right)\right\}=1 /\left(1+\alpha\left|w_{n}\right|^{2}\right)$, which is a one-sided Cauchy distribution with scale parameter $1 / \sqrt{\alpha}$.

\section{METHODS}

Two sets of data are investigated in this paper: a $128 \times 128$ SheppLogan phantom simulated for an 8-channel coil using the $B_{1}$ simulator available online at http://www. nmr.mgh.harvard.

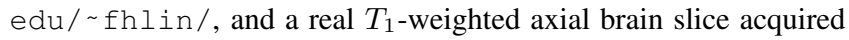
using a 3 -D MPRAGE sequence $(256 \times 256 \times 176$ sagittal slices, $1.0 \mathrm{~mm}$ isotropic resolution) with a 32 -channel Siemens head coil at $3 \mathrm{~T}$. The first data set is normalized to correct for non-uniform sumof-squares image intensity, and the second data set is split into axial slices, cropped and processed individually. The readout direction is perpendicular to the axial slice plane. The Shepp-Logan phantom is very sparse in the finite-differences domain, and the axial brain slices are compressible in the 'db2' DWT domain.

To measure the statistical mismatch of each penalty function for these two data sets, we perform a Kolmogorov-Smirnov (K-S) test, treating magnitudes of the sparse transform coefficients to be independent identically distributed (iid) random variables and computing the empirical cumulative distribution function (cdf) from those sparse coefficient magnitudes. We also plot the cdfs of the priors corresponding to the sparsity penalty functions against the empirical cdf of the data. Since the Welsch function has an improper associated prior, the cdf does not exist, and we leave out the Welsch function from this analysis. We perform this comparison first for the simulated data, and follow with the real data.

To complete the analysis, we take the both datasets, uniformly undersample them by a factor of 4 in both directions and run the SpRING algorithm for each sparsity penalty function on the undersampled data, using a central $(30 \times 30$ for the phantom, $36 \times 36$ for the brain) ACS block for determining the coil combination weights and GRAPPA kernel. The magnitude images of the results are compared against the reference magnitude images for SpRING with the $\ell_{1}$, Welsch, and Cauchy penalty functions. Also, reconstructions using the $\ell_{p}$-"norm" with continuation (start with $\alpha=1 / p=1$ and slowly increase $\alpha$ until $\alpha=1 / p=10$ ) are shown. 


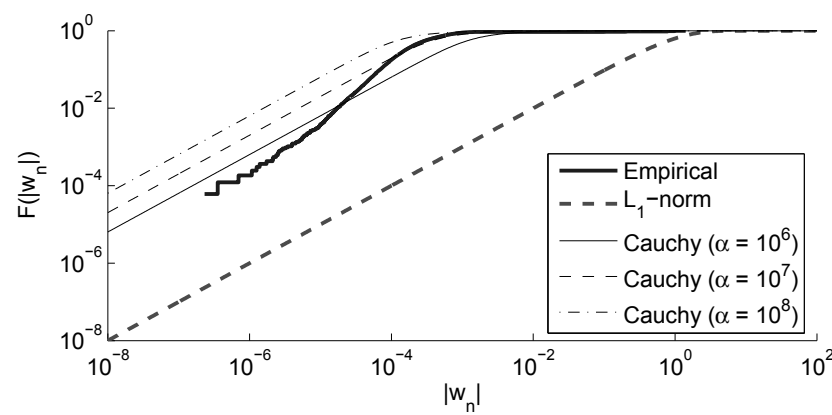

Fig. 1. The empirical cdf of the Shepp-Logan phantom coefficient magnitudes in the finite differences domain is plotted on a log-log scale. The cdfs of the sparsity priors for the $\ell_{1}$-norm and Cauchy penalty function for various choices of $\alpha$ are plotted against the empirical cdf.

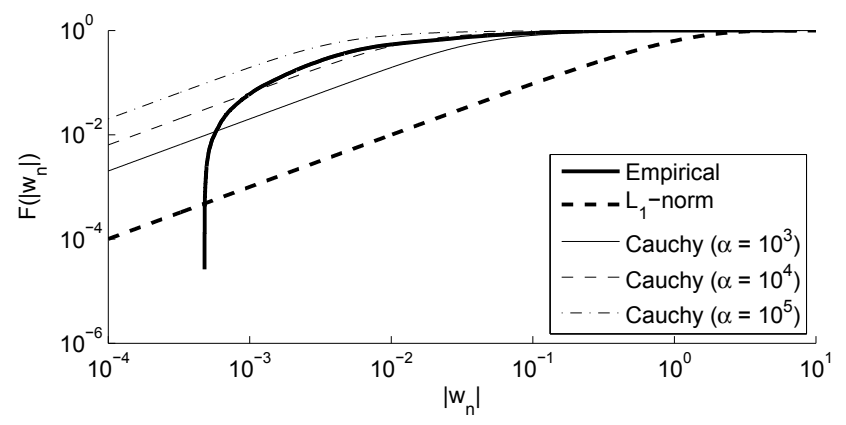

Fig. 2. The empirical cdf of the $T_{1}$-weighted MPRAGE brain slice coefficient magnitudes in the 'db2' DWT domain is plotted on a log$\log$ scale. The cdfs of the sparsity priors for the $\ell_{1}$-norm and Cauchy penalty function for various choices of $\alpha$ are plotted against the empirical cdf.

\section{RESULTS}

The Shepp-Logan phantom is very sparse in the finite differences domain, as confirmed by the empirical cdf of the sparse transform coefficient magnitudes shown in Figure 1. In Figure 1, the phantom is much more sparse than the prior corresponding to the $\ell_{1}$-norm, whereas the Cauchy function's cdf captures the behavior well for $\alpha$ in the neighborhood of $10^{6}$ or $10^{7}$. Evaluating the $\mathrm{K}-\mathrm{S}$ test statistic for the empirical data against these priors confirms what we observe in the figure; the test statistic ranges from 0.9316 for the $\ell_{1}$-norm prior to 0.09003 for the Cauchy penalty function with $\alpha=10^{7}$. Based on these results, we would not expect to capture all the sparsity of the Shepp-Logan phantom when performing a reconstruction using the $\ell_{1}$-norm or Cauchy penalty function with smaller $\alpha$, and we would expect over-smoothing to occur when using the Cauchy penalty function with $\alpha$ too large.

Unlike the Shepp-Logan phantom, real MR images are often only approximately sparse, and the 32 -channel $T_{1}$-weighted MPRAGE slice is one such example. To account for the additional coefficients in the sparse transform domain from padding the image to size $256 \times 256$ coefficients (the DWT implementation requires sizes that are powers of two), before computing the empirical cdf, the DWT coefficient magnitudes are sorted and the lowest values removed to reduce the total number of coefficients to $250 \times 170$, the size of the original image (zero-padding the image should not have introduced non-zero DWT coefficients, except maybe around the edges). Observing the empirical cdf shown in Figure 2, the best choice of $\alpha$ to model the empirical distribution with a Cauchy prior is $10^{4}$, three orders of smaller than a suitable choice of $\alpha$ for the very sparse Shepp-Logan phantom. For the real data, the $\ell_{1}$-norm prior has a $\mathrm{K}-\mathrm{S}$ statistic of 0.8008 , whereas the Cauchy prior has a $\mathrm{K}-\mathrm{S}$ statistic of 0.08928 . The difference between observed sparsity between the Shepp-Logan phantom and the real data accentuates the need to consider the nature of using real data when choosing an appropriate sparsity penalty function. Possibly, by choosing $\alpha$ too large, we are over-smoothing parts of the real image.

To illustrate the effects of choosing each sparsity penalty function on the SpRING reconstruction result, we run the SpRING algorithm with several different sparsity penalty functions on uniformly undersampled data over a coarse, then denser, set of $\lambda$ 's. First, we perform the reconstructions on the simulated Shepp-Logan phantom, where we observe significant differences between the results for different penalty functions. The results in Figure 3 suggest that SpRING with the Cauchy penalty function significantly outperforms SpRING using the other penalty functions. These results corroborate the interpretation of the $\mathrm{K}-\mathrm{S}$ statistics above that the Cauchy prior models the reference image better than the $\ell_{1}$-norm prior. The presence of significant artifacts in the $\ell_{1}$-norm, $\ell_{p}$-"norm", and Welsch function reconstructions suggest that those penalty functions do not model the Shepp-Logan phantom as well. The Welsch function also introduces numerical instability into the SpRING algorithm, as many of the non-sparse entries of the diagonal weighting matrix are treated as zero because of finite precision, which adversely affects its performance.

The results in Figure 4 illustrate the differences when regularizing the GRAPPA result with different sparsity priors. Although the $\ell_{p}$-"norm", Welsch and Cauchy functions perform similarly, the $\ell_{1}$-norm maintains the detail in the darker center region, whereas the other methods smooth the area to varying degrees. Conversely, SpRING using the Cauchy function de-noises the smooth regions of the image better than the other methods. Note in both cases the relative values of $\lambda$ that minimize the RMSE are lowest for the Welsch function, followed by the Cauchy penalty, $\ell_{1}$-norm, and $\ell_{p}$-"norm".

\section{CONCLUSION}

In conclusion, we observe important differences between synthetic and real data in choosing an appropriate sparsity penalty function. In both cases, the Cauchy function gives excellent performance, but the $\ell_{1}$-norm preserves the details better for the real data. Perhaps the Cauchy penalty function would perform better with a more representative sparse transform for the real data. Also, in the synthetic data case, the Welsch function is plagued by numerical issues, and both the $\ell_{1}$-norm and $\ell_{p}$-"norm" leave noticeable artifacts behind. In real data, the $\ell_{p}$-"norm", Welsch, and Cauchy functions oversmooth the image, while the $\ell_{1}$-norm achieves a better balance of increasing SNR and maintaining detail. The value of these local details makes the statistical analysis shown not as effective for choosing penalty functions for MR imaging. This behavior is consistent with the approximate sparsity of the real image. This work will be supplemented with similar testing for more sophisticated sparsifying transforms for the real data, to see if the results shift towards what is observed for simulated data. The reconstructions also will be repeated for random undersampling to understand how incoherence could affect the choice of sparsity penalty function. 


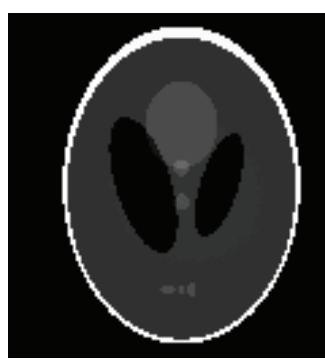

(a) Original image

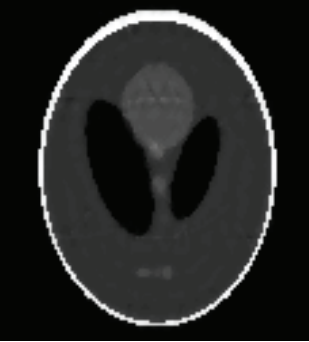

(b) SpRING with $\ell_{1}$-norm penalty $\lambda=10^{1.6}$ $\mathrm{RMSE}=0.01775$

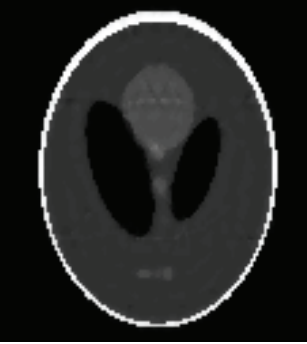

(c) SpRING with $\ell_{p}$-"norm" penalty $\lambda=10^{1.6} ; \alpha_{f}=10$ RMSE $=0.01708$

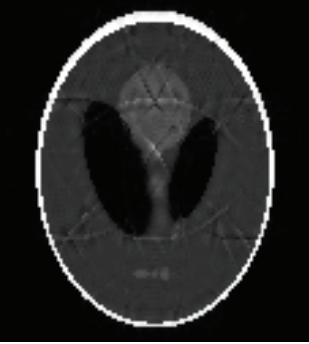

(d) SpRING with Welsch penalty

$\lambda=10^{0.2} ; \alpha_{f}=10^{6}$ RMSE $=0.02315$

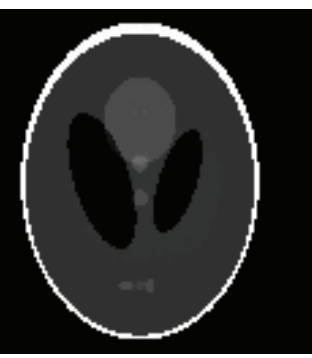

(e) SpRING with Cauchy penalty $\lambda=10^{1.4} ; \alpha_{f}=10^{7}$ RMSE $=0.002949$

Fig. 3. SpRING reconstructions using different sparsity penalty functions for the 8-channel simulated Shepp-Logan phantom. The last three methods use continuation; $\alpha_{f}$ refers to the final value of $\alpha$.

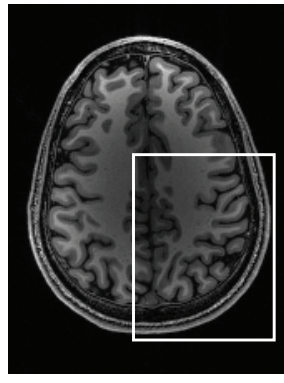

(a) Original image

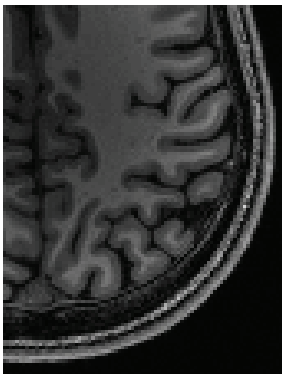

(b) Original image (inset)

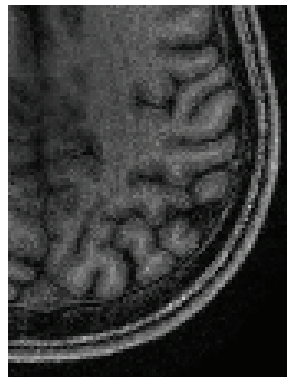

(c) SpRING with $\ell_{1}$-norm penalty $\lambda=10^{0.2}$

RMSE $=0.03591$

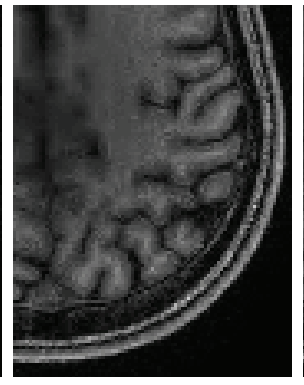

(d) SpRING with $\ell_{p}$ "“norm" penalty $\lambda=10^{0.4} ; \alpha_{f}=10$ RMSE $=0.03495$
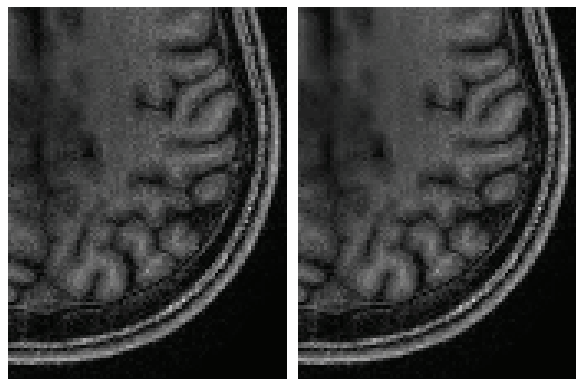

(e) SpRING with Welsch (f) SpRING with Cauchy $\lambda=10^{-0.4} ; \alpha_{f}=10^{6}$ penalty
$\lambda=10^{0} ; \alpha_{f}=10^{4}$
RMSE $=0.03487$

Fig. 4. SpRING reconstructions using different sparsity penalty functions for an axial slice of the 32 -channel $T_{1}$-weighted MPRAGE real data. The rectangle on the original image identifies the inset region portrayed for the different reconstructions. The last three methods use continuation; $\alpha_{f}$ refers to the final value of $\alpha$.

\section{REFERENCES}

[1] M. A. Griswold, P. M. Jakob, R. M. Heidemann, M. Nittka, V. Jellus, J. Wang, B. Kiefer, and A. Haase, "Generalized autocalibrating partially parallel acquisitions (GRAPPA)," Magn. Reson. Med., vol. 47, no. 6, pp. 1202-1210, Jun. 2002.

[2] E. J. Candès, J. Romberg, and T. Tao, "Robust uncertainty principles: Exact signal reconstruction from highly incomplete frequency information," IEEE Trans. Inf. Theory, vol. 52, no. 2, pp. 489-509, Feb. 2006.

[3] E. J. Candès and J. Romberg, "Quantitative robust uncertainty principles and optimally sparse decompositions," Found. Comput. Math., vol. 6, no. 2, pp. 227-254, Apr. 2006.

[4] D. L. Donoho, "Compressed sensing," IEEE Trans. Inf. Theory, vol. 52, no. 4, pp. 1289-1306, Apr. 2006.

[5] M. Lustig, D. Donoho, and J. M. Pauly, "Sparse MRI: The application of compressed sensing for rapid MR imaging," Magn. Reson. Med., vol. 58, no. 6, pp. 1182-1195, Dec. 2007.

[6] D. S. Weller, J. R. Polimeni, L. J. Grady, L. L. Wald, E. Adalsteinsson, and V. K. Goyal, "Combining nonconvex compressed sensing and GRAPPA using the nullspace method," in 18th Annual Meeting of ISMRM., May 2010, p. 4880.
[7] M. Lustig, M. Alley, S. S. Vasanawala, D. L. Donoho, and J. M. Pauly, "L $L_{1}$ SPIR-iT: Autocalibrating parallel imaging compressed sensing," in 17th Annual Meeting of ISMRM., Apr. 2009 , p. 379.

[8] L. Grady and J. R. Polimeni, "Nullspace compressed sensing for accelerated imaging," in 17th Annual Meeting of ISMRM., Apr. 2009, p. 2820.

[9] R. Chartrand, "Exact reconstruction of sparse signals via nonconvex minimization," IEEE Signal Process. Lett., vol. 14, no. 10, pp. 707-710, Oct. 2007.

[10] J. Trzasko and A. Manduca, "Highly undersampled magnetic resonance image reconstruction via homotopic $\ell_{0}$ minimization," IEEE Trans. Med. Imag., vol. 28, no. 1, pp. 106-121, Jan. 2009.

[11] P. B. Roemer, W. A. Edelstein, C. E. Hayes, S. P. Souza, and O. M. Mueller, "The NMR phased array," Magn. Reson. Med., vol. 16, no. 2, pp. 192-225, Nov. 1990.

[12] A. C. Zelinski, V. K. Goyal, and E. Adalsteinsson, "Simultaneously sparse solutions to linear inverse problems with multiple system matrices and a single observation vector," SIAM J. Sci. Comput., vol. 31, no. 6, pp. 4533-4579, Jan. 2010. 